\title{
Regulation of MT1-MMP and MMP-2 by the Serpin PEDF: a Promising New Target for Metastatic Cancer
}

\author{
Marice B. Alcantara Crispin R. Dass
}

School of Biomedical and Health Sciences, Victoria University, St Albans

\author{
Key Words \\ Cancer $\cdot \mathrm{PEDF} \cdot \mathrm{MT1}-\mathrm{MMP} \cdot \mathrm{MMP}-2 \cdot$ Collagen $・ \mathrm{MMP}-14 \cdot$ Metastasis
}

\begin{abstract}
The balance of endogenous angiogenic factors in the body is responsible for the homeostatic control of angiogenesis during normal physiological circumstances, with the disruption of this fragile balance leading to pathologic angiogenic events such as those involved in cancer progression. This review focuses regulation of the pro-angiogenic factors membrane type-1 matrix metalloproteinase (MT1-MMP) and matrix metalloproteinase-2 (MMP-2) by the antiangiogenic factor pigment epithelium-derived factor (PEDF) in the contexts of angiogenesis, cancer cell proliferation and metastasis. Understanding the role of PEDF in the regulation of MT1-MMP and MMP-2 as it pertains to cancer control is important in order to understand whether and how such associations provide a novel target for cancer therapy.
\end{abstract}

Copyright $(2013$ S. Karger AG, Basel

\section{Introduction}

Cancer is essentially the term that is used to describe a range of diseases characterized by uncontrolled cell proliferation and migration. Unrestrained cell growth results in the development of tumours, which may present themselves as benign or malignant. While benign tumours are characterised by encapsulated structures and a lack of metastatic 
behaviour, malignant tumours have the ability to invade and metastasize, an event modulated by the cancer cell and associated with an increased chance of morbidity [1].

Cancer cell growth is a complex process requiring several physiological changes to occur. Amongst these changes is angiogenesis, the proliferation and de novo alignment of new capillaries from pre-existing blood vessels, in order to support the growth of the tumour and its increased demand for oxygen and nutrients [2,3]. Under regular circumstances, the process of angiogenesis is regulated by the balance of pro- and anti-angiogenic factors, and occurs during normal healing and repair, however, several factors can result in angiogenesis associated diabetic retinopathy, arthritis, macular degeneration and also pro-metastastic tumour cell growth [3-6].

The role of angiogenesis in tumour cell growth has been widely investigated previously by numerous researchers, however, Folkman's group [2] were the ones who initially observed the existence of a correlation between angiogenesis and tumour growth, determining that the development of new blood vessels to supply nutrients and oxygen was necessary for the growth of the cancer cell, and in fact be crucial for metastasis via the bloodstream $[3,7]$.

In essence, the particular balance of pro- and anti-angiogenic factors regulates the process of angiogenesis. Such factors, which inhibit the angiogenic process, are pigment epithelium-derived factor (PEDF), thrombospondin-1 (TSP-1) and tissue inhibitor of metalloproteinases (TIMPs), whilst growth stimulators include vascular endothelial growth factors (VEGFs) and the matrix metalloproteinases (MMPs) [5, 6, 8].

Considering that the factors responsible for angiogenesis are sequestered within the extracellular matrix (ECM) and that cell growth occurs as a result of communication with the immediate environment, naturally, the ECM is essential to the process of both cancer cell growth and angiogenesis. The ECM consists of mainly three structures; (1) proteoglycans, (2) structural proteins that are largely composed of collagen I, II and III, and (3) proteins such as fibrillin and fibronectins [9]. In cancer, these components encounter extensive proteolytic degradation as the tumour cell grows and invades, with ECM degradation and vascular leakage both necessary for angiogenesis to occur and is a process which requires several enzymes, importantly matrix MMPs $[10,11]$.

As the name suggests, the MMP family of enzymes play an important role in the breakdown of the ECM [10]. Essentially, the MMP family are a group of calcium-and zincdependent enzymes $[10,12]$. Excluding MMP-7, MMP-26 and MMP-23, the typical MMP structure is composed of a hemopexin/vitronectin domain connected by a catalytic domain through a linker region [13]. In general, MMPs are produced by tissues as inactive zymogens and require further activation, however, membrane type matrix metalloproteinase-1 (MT1MMP; also known as MMP-14) does not require additional activation, due to its capacity to be presented on the cell membrane in its active form [14]. This is an integral part of MT1MMP's functions as it allows it to in turn, activate matrix metalloproteinase-2 (MMP-2) and combined, the co-ordinate actions of these two proteins have been correlated to the malignancy of a wide variety of cancers $[15,16]$.

As discussed, the role of MT1-MMP and MMP-2 in cancer is to facilitate the breakdown of the ECM to allow for aberrant tumour growth. In physiologic conditions, an anti-angiogenic compound balances the functions of pro-angiogenic factors, and one such anti-angiogenic factor found in the body is PEDF.

PEDF is a $50 \mathrm{kDa}$ secreted glycoprotein which was initially localized to the human foetal retinal pigment epithelium (RPE), where it was found to be secreted and to inhibit vascular growth in that tissue [17-19]. Essentially, PEDF has been determined to be the most potent endogenous angiogenic inhibitor in the body with the ability to prohibit neovascularization in the cornea, as well as endothelial cell growth, tumour proliferation and cancer metastasis $[17,20]$. Furthermore, PEDF has also been found to promote neuronal survival and cellular differentiation $[21,22]$.

Understanding the various functions of PEDF and the MMPs has been the focus of numerous separate studies previously, though little is known about the direct regulatory interactions that may exist between PEDF and MMPs. The MMP degradation of structural 


\begin{tabular}{|c|c|c|c|}
\hline & PEDF & & \\
\hline Functions & Pathway & Locations & \\
\hline Blocks MT1-MMP action & $\begin{array}{l}\text { Prevents MT1-MMP presentation on membrane } \\
\text { Facilitated by changes in RhoA/Rac1proportions }\end{array}$ & $\begin{array}{l}\text { Plasma membrane } \\
\text { ECM }\end{array}$ & \multirow{4}{*}{ [41] } \\
\hline Suppress tumour growth & Unspecified & $\begin{array}{l}\text { Tumour cells } \\
\text { ECM }\end{array}$ & \\
\hline Induces apoptosis & Blocks nuclear factor of T cells & $\begin{array}{l}\text { Endothelial cells } \\
\quad \text { ECM }\end{array}$ & \\
\hline Prohibits metastasis & $\begin{array}{l}\text { Amoeboid invasion restraint } \\
\text { Suppression of proteolytic activity in mesenchymal-like melanoma cells }\end{array}$ & ECM & \\
\hline Inhibits VEGF expression & Facilitate decreasedVEGF protein and mRNA synthesis & Human osteosarcoma cells & [35] \\
\hline Prohibits angiogenesis & Collagen-integrin binding & ECM proteins & [19] \\
\hline $\begin{array}{l}\text { Immunoprotective - defends } \\
\text { against oxidative stress }\end{array}$ & & In cells & \\
\hline Downregulates MMP-9 & $\begin{array}{l}\text { Exact pathway unclear- although nuclear factor kappa-B (NFKB) known to } \\
\text { mediate PEDF function }\end{array}$ & ECM & [18] \\
\hline $\begin{array}{l}\text { Upregulates: } \\
\text { - angiopoietin-2 } \\
\text {-TSP } 1 / 2\end{array}$ & & ECM & \\
\hline Bone synthesis and repair & The control of neovascularisation & $\begin{array}{l}\text { Osteoclast } \\
\text { Osteoblast } \\
\text { Growth plate }\end{array}$ & {$[23]$} \\
\hline
\end{tabular}

Table 1. PEDF's functions in the body and the molecular pathways underpinning these functions.

collagens in the ECM prior to angiogenesis, which are essential for structural stability and providing the anchoring points for molecules such as growth factors, fibronectins and proteoglycans, is implicated as an important precursor in cancer progression [10]. Conversely, PEDF has demonstrated capacity as an anti-angiogenesis factor and its ability to target only new vessel growth, as well as its neurotrophic properties and widespread availability in various tissues throughout the body, which indicate that it may be of use as a novel target for cancer therapy [23]. PEDF also has binding sites for collagen within its protein structure. This review will investigate the associations between PEDF, MT1-MMP and MMP-2 in angiogenesis and cancer metastasis.

\section{Understanding PEDF and its potential role in cancer therapy}

PEDF is a $50 \mathrm{kDa}$ endogenous protein found in the ECM belonging to the serpin (serine protease inhibitor) family, with a heparin- and collagen-binding site in its structure [18, 24$26]$. It is a potent neurotrophic and angiogenesis-inhibiting factor with tumour suppressor properties $[27,28]$.

It is within the ECM where PEDF (Table 1) is naturally found that its anti-angiogenic and tumour-suppressing qualities are understood to take place, however, the exact pathways in which PEDF exerts its various functions are yet to be elucidated $[19,29]$. Within the ECM, it has been reported that PEDF engages collagen type I (collagen-1) and III, with immediate binding to collagen-1 possible as a result of PEDF's negatively charged surface binding sites attraction for the positively charged domains of collagen-1 [30, 31]. In fact, PEDF's antiangiogenic effect is understood to be the result of its capacity to inhibit endothelial cell growth and migration through its interaction with collagen-1.

As discussed previously, collagen- 1 is a critical angiogenic scaffold essential to the development of capillaries. The interactions of PEDF and its collagen-1 binding site was 
investigated by in a study [30] which revealed the importance of this structure in PEDF's functions, determining that mutations in the collagen-1-binding site of PEDF are correlated to the increased progression of tumour vasculature, which consequently allows for tumour advancement.

In addition to PEDF's collagen-1 binding site, its anti-angiogenic functions may be the result of its ability to reduce vessel permeability resulting from VEGF stimulation, by modulating receptors on the cell's surface and inducing apoptosis $[19,30]$. Interestingly, it has also been suggested that similar to the pro-angiogenic factors such as VEGF and the proteases of the MMP family, PEDF may exert its anti-angiogenic actions on ECM factors, especially collagen I-IV with its highest affinity reserved for collagen-1 [30-32].

It is through the ability to prohibit angiogenesis that PEDF's possible anti-tumour effects were initially examined, in osteosarcoma (OS) research especially. Previous research has shown that PEDF expression levels especially concentrated in areas of the growth plate of bone is correlated with a resistance to tumour growth in that same area [21]. Furthermore, in addition to its anti-angiogenic functions, there are other various ways in which PEDF is understood to attain its anti-tumour effects. These include its ability to inhibit tumour cell migration by promoting cell adhesion, its ability to induce cellular apoptosis and its ability to regulate the differentiation of tumour cells [25]. In effect, a decrease in PEDF concentrations has been correlated to an increased chance of metastasis and poor differentiation in prostate cancer [33].

However, despite the widely reported anti-angiogenic properties of PEDF, when the role of PEDF on choroidal neovascularisation (CNV), endothelial cell migration and vascular tube formation in vivo was evaluated, it was found that PEDF's anti-angiogenic activities were dependent on the concentration dose of protein that was administered [34]. Remarkably, it was found that although PEDF exerted a decrease in neovascularisation at low concentrations $(90 \mu \mathrm{g} / \mathrm{ml}$ ), a large increase in PEDF concentrations (to $360 \mu \mathrm{g} / \mathrm{ml}$ ) paradoxically resulted in the promotion of vascular growth [34]. The study also found that contrary to previous research, PEDF was able to stimulate VEGF and endothelial cells, which led to an increase in vessel formation. However, the results of the study may have been the result of the regulatory interactions between the pro- and anti-angiogenic factors, and consistent with homeostatic regulation, the large increase in PEDF may have stimulated increases in VEGF and vessel growth.

In various types of tumour cells, PEDF has been shown to decrease levels of VEGF [3538]. This is in line with increasing understanding of the complex cross-regulatory interactive nature of molecular components of normal tissue and especially tumour micromilieu in the past decade of cell and molecular biological research. However, despite PEDF's potent antiangiogenic and tumour effects, PEDF's exact molecular mechanisms remain to be completely understood [39]. One perhaps enigmatic molecular function of PEDF is its relationship with MT1-MMP and MMP-2.

\section{The regulatory interactions between PEDF, MT1-MMP and MMP-2}

As previously discussed, MT1-MMP is a member of the MMP family of proteases which has been implicated in various cancers for its matrix degrading and pro-angiogenic capabilities [16]. In contrast, PEDF is a protein belonging to the serpin family of inhibitors, which exerts various functions, but importantly, has anti-angiogenic and anti-tumour capabilities [28]. There has been extensive research undertaken which focuses on these two proteins individually, and how their functions affect the outcome of a variety of cancers. However, there have been very few studies which have investigated the direct relationship of these two proteins, and those that are currently available only briefly discuss their associations.

Currently, the interaction between PEDF and MT1-MMP was initially reported in a study in which treatment of chondrosarcoma cells with PEDF resulting in the decrease of MT1MMP concentrations [40]. More recently [41], in melanoma cells, increased levels of PEDF 
Fig. 1. The direct regulatory interactions between PEDF, MT1MMP and MMP-2, including PEDF's ability to downregulate MT1-MMP, MT1-MMP's role in activating MMP-2 and MMP-2's ability to downregulate PEDF.

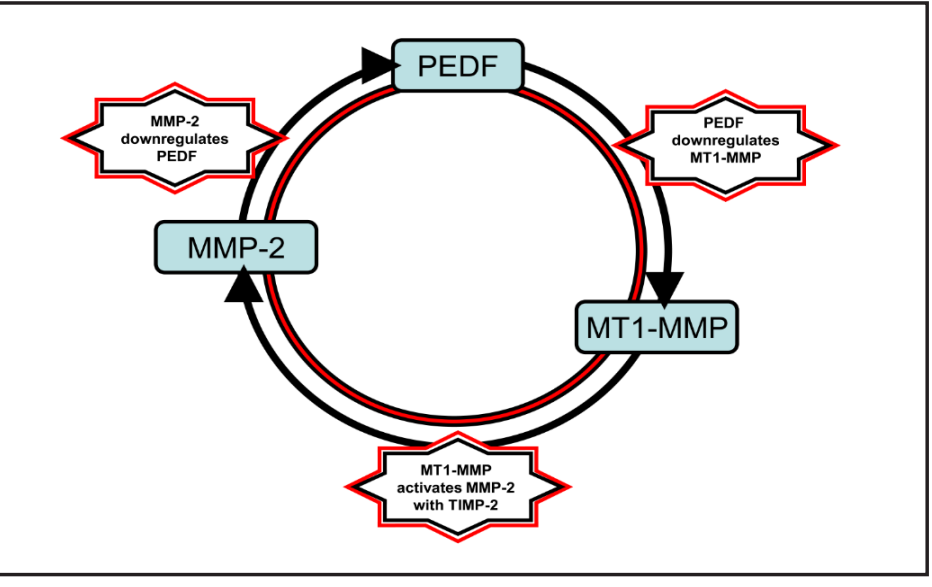

resulted in the prevention of the presentation of the MT1-MMP molecule on the cell surface.

MMP-2 is a protease which is closely associated with MT1-MMP (and the malignancy of several cancer types) which interestingly, has been found to be closely associated to PEDF, and MMP-2 has been implicated to downregulate PEDF [42]. The interactions between MMP2 and PEDF were further demonstrated in a more recent study by [43] which identified that PEDF-deficient mice models with pancreatic tumours displayed increased levels of the proteins MMP-2 and/or MMP-9. Therefore, these findings indicate an inverse correlation between PEDF and MMP-2 during hypoxia and pancreatic cancer, which we hypothesize to occur via MT1-MMP and its activation of MMP-2, though further studies are warranted to confirm this hypothesis.

Given that previous research have suggested the role of PEDF in downregulating MT1MMP, then therefore, as a result of MMP-2's reliance on MT1-MMP for activation (Fig. 1), PEDF may also possess the ability to control MMP-2 concentrations despite MMP-2's reported ability to downregulate PEDF concentration levels (though this has to be proven in cells). As a result, PEDF's functions may extend to its ability to regulate MT1-MMP and MMP2 , two of the common proteases implicated in metastatic cancer.

Two recent studies have already shown the efficacy due to systemic delivery of PEDF protein or peptides in models of tumours in vivo [25, 44]. In both cases, the effects of the protein were more significant when metastasis was measured rather than growth of the primary tumour. It is almost common knowledge that it is the spread of a cancer that places the life of the patient in jeopardy, as primary tumours are usually resectable or responsive to therapy. This therefore, open up the promising window of opportunity of being able to curb the spread of tumours with PEDF. As hinted to above, the authors speculate that such important molecular players dictating the ability of cancer cells to migrate from one site to another as MMP-2 and MT1-MMP may be under the control of PEDF.

\section{Conclusion}

Cancer progression is associated with angiogenesis, cell migration, and metastasis, events which are modulated by members of the MMP family of proteases, including MT1-MMP and MMP-2. In contrast to the roles of the MMPs, the functions of the potent antiangiogenic protein PEDF in cancer are varied, but essentially include its ability to prevent angiogenesis and tumour progression. Although the molecular mechanisms underpinning PEDF's anticancer activities have yet to be completely understood, it has shown its effectiveness against a variety of cancers. The role of PEDF has been investigated by directly focusing on its capacity to prevent cancer cell growth and angiogenesis in vitro and in vivo. We highlight another aspect of PEDF that warrants further studies: PEDF's associations with the critical factors of angiogenesis, MT1-MMP and MMP-2. 
Alcantara/Dass: MMP-2 and MMP-14 Regulation by PEDF

\section{Expert opinion}

Several studies have indicated the interactions between MT1-MMP and MMP-2 in the progression and malignancy of various cancer types, and suggest the inhibition of these MMPs to be important endeavors for exploration in oncological therapy. While the roles of PEDF, MT1-MMP and MMP-2 in various cancers has been individually researched previously and widely reported, little is known about the associations which exist between the three proteins, certainly for cancer progression, and how this knowledge may be utilised in cancer therapy. All that is known is PEDF negates MT1-MMP activation and therefore its functions associated with malignant cancer promotion. The antagonistic role of PEDF towards MT1MMP action further affects the latter's ability to activate and coordinate its actions with MMP-2, indicating that PEDF may be therapeutically effective in preventing the metastasis and malignancy associated with MT1-MMP and MMP-2 engagement. It would be worthwhile to investigate the interactions which exist directly between these three proteins, which individually are, as previous studies have shown, critical markers in cancer progression. Subsequently, future research should be directed at investigating how PEDF may be utilised towards regulating expression of proteins that are responsible for the process of angiogenesis, cancer cell growth, intravasion and metastasis, essentially, as we outline, via its effects on MT1-MMP and MMP-2.

\section{Conflict of interest}

The authors declare no conflict of interest in writing this article.

\section{References}

1 La Rocca G, Pucci-Minafra I, Marrazzo A, Taormina P, Minafra S: Zymographic detection and clinical correlations of MMP-2 and MMP-9 in breast cancer sera. Br J Cancer 2004;90:1414-1421.

- Folkman J: Tumor angiogenesis: therapeutic implications. N Engl J Med 1971;285:1182-1186.

3 Zetter BR: Angiogenesis and tumor metastasis. Annu Rev Med 1998;49:407-424.

4 Dass CR, Tran TM, Choong PF: Angiogenesis inhibitors and the need for anti-angiogenic therapeutics. J Dent Res 2007;86:927-936.

5 Aparicio S, Sawant S, Lara N, Barnstable CJ, Tombran-Tink J: Expression of angiogenesis factors in human umbilical vein endothelial cells and their regulation by PEDF. Biochem Biophys Res Commun 2005;326:387-394.

6 Ribatti D: Endogenous inhibitors of angiogenesis: a historical review. Leuk Res 2009;33:638-644.

7 Pang RWC, Poon RTP: Clinical implications of angiogenesis in cancers. Vasc Health Risk Manag 2006;2:97108.

$>8$ Liekens S, De Clercq E, Neyts J: Angiogenesis: regulators and clinical applications. Biochem Pharmacol 2001;61:253-270.

9 Karagiannis ED, Popel AS: A theoretical model of type I collagen proteolysis by matrix metalloproteinase (MMP) 2 and membrane type $1 \mathrm{MMP}$ in the presence of tissue inhibitor of metalloproteinase 2. J Biol Chem 2004;279:39105-39114.

10 Sounni NE, Paye A, Host L, Noël A: MT-MMPS as regulators of vessel stability associated with angiogenesis. Front Pharmacol 2011;2:111.

11 Gialeli C, Theocharis AD, Karamanos NK: Roles of matrix metalloproteinases in cancer progression and their pharmacological targeting. FEBS J 2011;278:16-27.

12 Hadler-Olsen E, Fadnes B, Sylte I, Uhlin-Hansen L, Winberg JO: Regulation of matrix metalloproteinase activity in health and disease. FEBS J 2011;278:28-45. 


\section{Cellular Physiology Cell Physiol Biochem 2013;31:487-494 and Biochemistry

13 Sternlicht MD, Werb Z: How matrix metalloproteinases regulate cell behavior. Annu Rev Cell Dev Biol 2001;17:463-516.

14 Itoh Y, Seiki M: MT1-MMP: a potent modifier of pericellular microenvironment. J Cell Physiol 2006;206:1-8.

15 Sato H, Takino T: Coordinate action of membrane-type matrix metalloproteinase-1 (MT1-MMP) and MMP-2 enhances pericellular proteolysis and invasion. Cancer Sci 2010;101:843-847.

16 Al-Raawi D, Abu-El-Zahab H, El-Shinawi M, Mohamed MM: Membrane type-1 matrix metalloproteinase (MT1-MMP) correlates with the expression and activation of matrix metalloproteinase-2 (MMP-2) in inflammatory breast cancer. Int J Clin Exp Med 2011;4:265-275.

17 Becerra SP, Fariss RN, Wu YQ Montuenga LM, Wong P, Pfeffer BA: Pigment epithelium-derived factor in the monkey retinal pigment epithelium and interphotoreceptor matrix: apical secretion and distribution. Exp Eye Res 2004;78:223-234.

18 Filleur S, Nelius T, de Riese W, Kennedy RC: Characterization of PEDF: a multi-functional serpin family protein. J Cell Biochem 2009;106:769-775.

19 Ren JG, Jie C, Talbot C: How PEDF prevents angiogenesis: a hypothesized pathway. Med Hypotheses 2005;64:74-78.

20 Tan ML, Choong PF, Dass CR: Anti-chondrosarcoma effects of PEDF mediated via molecules important to apoptosis, cell cycling, adhesion and invasion. Biochem Biophys Res Commun 2010;398:613-618.

21 Ek ET, Contreras KG, Dass CR, Choong PF: Inhibition of orthotopic osteosarcoma growth and metastasis by multitargeted antitumor activities of pigment epithelium-derived factor. Clin Exp Metastasis 2007;24:93106.

22 Mirochnik Y, Aurora A, Schulze-Hoepfner FT, Deabes A, Shifrin V, Beckmann R, Polsky C, Volpert OV: Short pigment epithelial-derived factor-derived peptide inhibits angiogenesis and tumor growth. Clin Cancer Res 2009;15:1655-1663.

23 Chandolu V, Dass CR: Cell and molecular biology underpinning the effects of PEDF on cancers in general and osteosarcoma in particular. J Biomed Biotechnol 2012;2012:740295.

24 Setten GV, Abdiu O: Possible role of pigment epithelium derived factor in angiogenesis. European Opthalmic Rev 2009:10:64-67.

25 Yasui N, Mori T, Morito D, Matsushita O, Kourai H, Nagata K, Koide T: Dual-site recognition of different extracellular matrix components by antiangiogenic/ neurotrophic serpin, PEDF. Biochemistry 2003;42:3160-3167.

26 Kawaguchi T, Yamagishi SI, Sata M: Structure-function relationships of PEDF. Curr Mol Med 2010;10:302311.

27 Wu YQ Becerra SP: Proteolytic activity directed toward pigment epithelium-derived factor in vitreous of bovine eyes. Implications of proteolytic processing. Invest Ophthalmol Vis Sci 1996;37:1984-1993.

28 Broadhead ML, Dass CR, Choong PF: Cancer cell apoptotic pathways mediated by PEDF: prospects for therapy. Trends Mol Med 2009;15:461-467.

29 Subramanian P, Deshpande M, Locatelli-Hoops S, Moghaddam-Taaheri S, Gutierrez D, Fitzgerald DP, Guerrier S, Rapp M, Notario V, Becerra SP: Identification of pigment epithelium-derived factor protein forms with distinct activities on tumor cell lines. J Biomed Biotechnol 2012;2012:425907.

- 30 Hosomichi J, Yasui N, Koide T, Soma K, Morita I: Involvement of the collagen I-binding motif in the antiangiogenic activity of pigment epithelium-derived factor. Biochem Biophys Res Commun 2005;335:756761.

31 Meyer C, Notari L, Becerra SP: Mapping the type I collagen-binding site on pigment epithelium-derived factor. Implications for its antiangiogenic activity. J Biol Chem 2002;277:45400-45407.

32 Quan GM, Ojaimi J, Li Y, Kartsogiannis V, Zhou H, Choong PF: Localization of pigment epithelium-derived factor in growing mouse bone. Calcif Tissue Int 2005;76:146-153.

-33 Halin S, Wikström P, Rudolfsson SH, Stattin P, Doll JA, Crawford SE, Bergh A: Decreased pigment epitheliumderived factor is associated with metastatic phenotype in human and rat prostate tumors. Cancer Res 2004;64:5664-5671.

-34 Apte RS, Barreiro RA, Duh E, Volpert O, Ferguson TA: Stimulation of neovascularization by the antiangiogenic factor PEDF. Invest Ophthalmol Vis Sci 2004;45:4491-4497.

-35 Takenaka K, Yamagishi S, Jinnouchi Y, Nakamura K, Matsui T, Imaizumi T: Pigment epithelium-derived factor (PEDF)-induced apoptosis and inhibition of vascular endothelial growth factor (VEGF) expression in MG63 human osteosarcoma cells. Life Sci 2005;77:3231-3241. 
-36 Ek ET, Contreras KG, Dass CR, Choong PF: PEDF-derived synthetic peptides exhibit antitumor activity in an orthotopic model of human osteosarcoma. J Orthop Res 2007;25:1671-1680.

37 Yang J, Chen S, Huang X, Han J, Wang Q Shi D, Cheng R, Gao G, Yang X: Growth suppression of cervical carcinoma by pigment epithelium-derived factor via anti-angiogenesis. Cancer Biol Ther 2010;9:967-974.

-38 Zhang Y, Han J, Yang X, Shao C, Xu Z, Cheng R, Cai W, Ma J, Yang Z, Gao G: Pigment epithelium-derived factor inhibits angiogenesis and growth of gastric carcinoma by down-regulation of VEGF. Oncol Rep 2011;26:681-686.

-39 Manalo KB, Choong PF, Dass CR: Pigment epithelium-derived factor as an impending therapeutic agent against vascular epithelial growth factor-driven tumor-angiogenesis. Mol Carcinog 2011;50:67-72.

40 Tan ML, Choong PF, Dass CR: Anti-chondrosarcoma effects of PEDF mediated via molecules important to apoptosis, cell cycling, adhesion and invasion. Biochem Biophys Res Commun 2010;398:613-618.

41 Ladhani O, Sánchez-Martinez C, Orgaz JL, Jimenez B, Volpert OV: Pigment epithelium-derived factor blocks tumor extravasation by suppressing amoeboid morphology and mesenchymal proteolysis. Neoplasia 2011;13:633-642.

42 Notari L, Miller A, Martínez A, Amaral J, Ju M, Robinson G, Smith LE, Becerra SP: Pigment epitheliumderived factor is a substrate for matrix metalloproteinase type 2 and type 9: implications for downregulation in hypoxia. Invest Ophthalmol Vis Sci 2005;46:2736-2747.

43 Grippo PJ, Fitchev PS, Bentrem DJ, Melstrom LG, Dangi-Garimella S, Krantz SB, Heiferman MJ, Chung C, Adrian K, Cornwell ML, Flesche JB, Rao SM, Talamonti MS, Munshi HG, Crawford SE: Concurrent PEDF deficiency and Kras mutation induce invasive pancreatic cancer and adipose-rich stroma in mice. Gut 2012;61:1454-1464.

44 Broadhead ML, Choong PF, Dass CR: Efficacy of continuously administered PEDF-derived synthetic peptides against osteosarcoma growth and metastasis. J Biomed Biotechnol 2012;2012:230298. 